\title{
Tumor suppressor ING4 inhibits estrogen receptor activity in breast cancer cells
}

\author{
This article was published in the following Dove Press journal: \\ Breast Cancer - Targets and Therapy \\ 17 November 2016 \\ Number of times this article has been viewed
}

\author{
Madeline M Keenen' \\ Suwon $\mathrm{Kim}^{1,2}$ \\ 'Department of Basic Medical \\ Sciences, University of Arizona \\ College of Medicine - Phoenix, \\ Phoenix, AZ, ${ }^{2}$ Division of Cancer and \\ Cell Biology, Translational Genomics \\ Research Institute, Phoenix, AZ, USA
}

Correspondence: Suwon Kim Department of Basic Medical Sciences, University of Arizona College of Medicine Phoenix, 425 North 5th Street, Phoenix, AZ 85004, USA

Tel + I 6023438762

Email suwon@email.arizona.edu

\begin{abstract}
Resistance to antiestrogen therapy remains a significant problem in breast cancer. Low expression of inhibitor of growth 4 (ING4) in primary tumors has been correlated with increased rates of recurrence in estrogen receptor-positive $(\mathrm{ER}+)$ breast cancer patients, suggesting a role for ING4 in ER signaling. This study provides evidence that ING4 inhibits ER activity. ING4 overexpression increased the sensitivity of T47D and MCF7 ER+ breast cancer cells to hormone deprivation. ING4 attenuated maximal estrogen-dependent cell growth without affecting the dose-response of estrogen. These results indicated that ING4 functions as a noncompetitive inhibitor of estrogen signaling and may inhibit estrogen-independent ER activity. Supportive of this, treatment with fulvestrant but not tamoxifen rendered T47D cells sensitive to hormone deprivation as did ING4 overexpression. ING4 did not affect nuclear ER $\alpha$ protein expression, but repressed selective ER-target gene transcription. Taken together, these results demonstrated that ING4 inhibited estrogen-independent ER activity, suggesting that ING4-low breast tumors recur faster due to estrogen-independent ER activity that renders tamoxifen less effective. This study puts forth fulvestrant as a proposed therapy choice for patients with ING4-low ER+ breast tumors. Keywords: tamoxifen resistance, transcription repression, PDZK1, TFF1, estrogen independent ERa, fulvestrant
\end{abstract}

\section{Introduction}

Estrogen receptor (ER) signaling plays a pivotal role in breast cancer and has farreaching therapeutic implications as $>70 \%$ of breast tumors express ER. ${ }^{1}$ ER is best characterized as a transcription regulator activated by its ligand estrogen binding, which in turn binds directly or indirectly to DNA in the genome and modulates gene expression involved in normal cell function and tumor progression. ${ }^{2,3}$ Limiting estrogen binding to ER has been a successful strategy to treat ER-positive $(\mathrm{ER}+)$ breast cancer, specifically in an adjuvant setting for the prevention of tumor recurrence. Selective ER modulators (SERMs; eg, tamoxifen) that compete with estrogen for binding to ER have proven effective in reducing tumor recurrence and mortality in ER+ breast cancer patients by $50 \%$ and $30 \%$, respectively. ${ }^{4}$ Newer generation antiestrogen agents, aromatase inhibitors (AIs; eg, anastrozole) that inhibit estrogen production in peripheral tissue, have demonstrated increased efficacy in reducing tumor recurrence in postmenopausal ER+ breast cancer patients. ${ }^{5}$ These study outcomes have warranted antiestrogen agents as the staple therapy in ER+ breast cancer treatment. However, it has long been recognized that $2 \%-5 \%$ of patients relapse each year even during therapy, indicating that a significant portion of breast tumors have intrinsic resistance to antiestrogen therapy. ${ }^{5}$ 
The mechanisms of antiestrogen therapy resistance have been extensively investigated using cell models subjected to long-term hormone deprivation or tamoxifen treatment. One of the converging mechanisms from these studies is aberrant growth factor (GF) signaling involving receptor kinases such as epidermal growth factor receptor (EGFR), ERBB2/HER2, insulin-like growth factor receptor (IGF1R), and fibroblast growth factor receptor 1 (FGFR1), and their downstream molecules such as PI3K/AKT, MAPK, and MYC..$^{6-12}$ GF signaling not only promotes tumor cell proliferation and survival but also directly activates ER through phosphorylation. ${ }^{6} \mathrm{GF}$-activated ER $\alpha$ was shown to bind to the genomic regions distinct from estrogen-induced ER $\alpha$ binding sites and induces gene expression related to endocrine therapy resistance. ${ }^{13}$ Supportive of the notion that GF signaling plays a significant role in aggressive breast cancer and/or therapy resistance, breast tumor samples from patients who do not benefit from therapy often harbor mutations or activation signatures related to the GF signaling pathways. ${ }^{14}$ Not only does GF signaling activate ER, but a subset of estrogenbound ER localized in the cytoplasm has also been shown to activate GF signaling by directly associating with GF receptors or with GF signaling components, underscoring the bidirectional crosstalk between ER and GF signaling in breast cancer. ${ }^{6}$ However, combination therapy using antiestrogen and GF pathway-targeting agents has not been successful in improving breast cancer outcome in clinical trials, presenting challenges to the strategies to counter GF signaling-related endocrine therapy resistance in breast cancer. ${ }^{15-17}$ The fact that GF signaling pathway mutations have been found in tumors resistant to cancer therapeutics not related to antiestrogen agents may suggest that GF signaling may provide therapy resistance in general by providing tumor cell growth and survival advantage, rather than conferring resistance specific to antiestrogen therapy. ${ }^{18}$

The more direct mechanism of antiestrogen therapy resistance is mutation of the ER encoding gene, ESR 1 , found in $20 \%-50 \%$ of recurrent tumors in patients treated with adjuvant SERM and/or AI therapy. ${ }^{19,20}$ These mutations result in an ER protein conformation that mimics the estrogenbound state of ER, negating the requirement for estrogen and rendering antiestrogen agents ineffective. ${ }^{21}$ However, the ESR 1 mutations are found at very low frequencies in primary tumors, suggesting that these mutations are likely to represent acquired resistance under selective pressure of antiestrogen therapy. ${ }^{21,22}$ Thus, the ESRI mutation status has limited utility as a diagnostic marker and/or therapy target for the antiestrogen therapy resistance that plagues patients during the initial stages of breast cancer treatment.
Several gene expression signatures associated with poor prognosis related to tamoxifen or AI resistance have emerged, some of which are clinically available as prognostic tests. ${ }^{14}$ However, the variability between the gene signatures may attest to the heterogeneity of intrinsic antiestrogen resistance and/or the diversity of technical and computational platforms used in deriving each gene signature. Clinical utility of the gene signatures to predict resistance to antiestrogen therapy awaits reports from ongoing trials. ${ }^{14}$ As such, a need to better understand genetic factors that determine intrinsic antiestrogen therapy resistance still remains.

Inhibitor of growth 4 (ING4) is a member of the ING tumor suppressor family (ING1-5) that regulates histone modification and gene transcription. ${ }^{23}$ It has been shown that the ING4 gene is deleted in $16 \%$ or downregulated in $34 \%$ of breast tumors. ${ }^{24,25}$ Low expression of ING4 was correlated with advanced tumor features and lymph node positivity, suggesting that downregulation of ING4 may contribute to breast cancer progression. ${ }^{25}$ More clinically relevant, patients with ING4-low expressing primary tumors relapsed at a faster rate. In particular, ING4-low expression was associated with more than three times the recurrence rate in a cohort of ER+ breast cancer patients who were treated with adjuvant tamoxifen. ${ }^{25}$ These results raised a question whether ING4 played a role in ER signaling and/or tamoxifen response. This study investigated a functional relationship between ING4 and ER in breast cancer cells. The results demonstrate that ING4 inhibits ligand-independent ER activity in the nucleus that allows growth of ER+ breast cancer cells in the absence of estrogen. These results suggest that ING4-low tumors contain unregulated ligand-independent ER activity, which renders tamoxifen less effective in patients. This study proposes downregulation of ING4 as a mechanism of intrinsic antiestrogen therapy resistance in ER+ breast cancer.

\section{Materials and methods Cell culture and reagents}

T47D and MCF7 cells that express the retroviral vector pMIG or the pMIG-based ING4 overexpression construct have been previously described. ${ }^{25,26}$ T47D and MCF7 cells were grown in the Roswell Park Memorial Institute (RPMI) and Minimum Essential Medium with Earle's Balanced Salt Solution (MEM/EBSS) media (Hyclone, Logan, UT, USA), respectively, containing $10 \%$ fetal bovine serum (FBS, Hyclone) and $10 \mu \mathrm{g} / \mathrm{mL}$ human insulin (Sigma-Aldrich, St. Louis, MO, USA). For hormone deprivation, cells were grown in respective phenol red free media (Invitrogen, Carlsbad, CA, USA) containing 10\% charcoal-stripped FBS (Hyclone). The reagents $17 \beta$-estradiol (E2, Sigma) and 
ICI182,780 (Sigma) were dissolved in dimethyl sulfoxide (DMSO), and 4-hydroxy tamoxifen (OHT, Sigma) was dissolved in $100 \%$ ethanol.

\section{In vitro cell proliferation assay}

Cells were plated at a density of 2,000 cells per well in 96-well plates in triplicate wells. Cells were grown in various media conditions for 7-14 days. Cells were fixed with $10 \%$ trichloroacetic acid followed by sulforhodamine B (SRB) colorimetric assay to measure relative cell numbers as described previously. ${ }^{25}$ Cell growth assays were repeated in three or more independent experiments.

\section{Western blot analysis}

Cell lysates were fractionated by lysing cells in a hypotonic buffer $(10 \mathrm{mM}$ Tris $\mathrm{pH} 8,10 \mathrm{mM} \mathrm{NaCl}, 0.2 \%$ Nonidet $\mathrm{P}-40$ ) on ice for $5 \mathrm{~min}$, followed by centrifugation at $1,800 \times g$ for $5 \mathrm{~min}$ to collect nuclei and cytoplasm. Nuclei were lysed in radioimmunoprecipitation assay (RIPA) buffer followed by sonication. Nuclear and cytoplasmic fractions were analyzed by Western blot using antibodies against ER $\alpha$ (Cell Signaling, Danvers, MA, USA), ING4 (EMD Millipore, Temecula, CA, USA), histone H3 (Cell Signaling), and tubulin (Cell Signaling), and phosphoextracellular signal-regulated kinase (ERK) (Cell Signaling) at 1:1,000 dilution.

\section{Luciferase assay}

The luciferase reporter plasmid, 3xERE-TATA-luc, was purchased from Addgene (Cambridge, MA, USA). ${ }^{27}$ T47DpMIG or T47D-ING4 cells were co-transfected with the linearized luciferase reporter plasmid and a neomycin resistance gene containing plasmid, pLNCx (Clontech, Mountain View, CA, USA) using Effectene (Qiagen Valencia, CA, USA) and were selected in the media containing $400 \mathrm{mg} /$ mL Geneticin (Gibco, Billings, MT, USA). Cells plated at $50 \%$ confluency in a $24-w e l l$ dish were hormone-deprived in the media containing $10 \%$ of charcoal-stripped FBS for $48 \mathrm{~h}$ and with or without $100 \mathrm{nM} \mathrm{E} 2$ for additional $24 \mathrm{~h}$. Luciferase activity in $30 \mu \mathrm{L}$ of cell lysates was measured using a Steady-Glo Luciferase Assay System (Promega Corporation, Fitchburg, WI, USA) and Victor3 luminometer (Perkin Elmer Life Sciences Products, Boston, MA, USA). Total protein concentration in cell lysates was measured using the BCA Protein Assay Kit (Pierce Thermo Fisher Scientific, Waltham, MA, USA). Luciferase activity was calculated as relative light units per microgram of protein and normalized to the $l u c 2$ gene copy number integrated into the genome as described previously. ${ }^{25}$

\section{Quantitative reverse transcription polymerase chain reaction ( $\mathrm{QRTPCR}$ )}

Total RNA was isolated from $5 \times 10^{5}$ cells using the RNeasy mini kit (Qiagen). Complementary DNA was synthesized from $2-5 \mu \mathrm{g}$ of total RNA using the Superscript III First-Strand Synthesis SuperMix kit with oligo dT primers (Invitrogen). Quantitative PCR was performed using Taqman Gene Expression Assays (Applied Biosystems, Foster City, CA, USA) with FAM-labeled probes for PDZK1, TFF1, ESR 1, PGR, and MYC, and VIC-labeled probe for GAPDH. Reactions were run using the Applied Biosystems 7900HT (Applied Biosystems) or StudioQuant 6 Real-Time PCR instrument (Life Technology, Carlsbad, CA, USA). Data were analyzed by the $\Delta \Delta \mathrm{C}_{\mathrm{t}}$ method normalized to $G A P D H$.

\section{Statistical analysis}

Unpaired 2-tailed Student's $t$-test was used to determine the statistical significance between two experimental number sets. Estrogen dose-response curve and half-maximal effective concentration (EC50) values were generated by nonlinear regression analysis with variable slope using GraphPad Prism software version v6.0 (GraphPad Software, Inc., La Jolla, CA, USA). Extra sum of squares $F$-test was used to determine a statistical significance of the differences between the two curves.

\section{Results \\ ING4 overexpression increases sensitivity of ER+ breast cancer cells to hormone deprivation and to tamoxifen treatment}

To assess the effects of ING4 on estrogen signaling, ING4 was overexpressed in two ER+ breast cancer cell lines, T47D and MCF7, using the retroviral vector $\mathrm{pMIG}$ as described previously. ${ }^{25}$ The growth between the vector (pMIG) and ING4 overexpressing cells was compared in the media containing full serum (FS) or in the hormone-deprived media containing charcoal-stripped serum (CSS) with or without E2. The ability of OHT blocking E2-depedent cell growth was also assessed in the same assay. The results showed that ING4 cells grew with a comparable rate to pMIG cells in FS, as has been reported previously; ${ }^{25,26} \mathrm{pMIG}$ or ING4 cells increased cell numbers by $200 \%-300 \%$ in 7 days (Figure $1 \mathrm{~A}$ and $\mathrm{B}$ ). In CSS, pMIG cells grew by $\leq 50 \%$ in 7 days (Figure 1A and B), demonstrating that both T47D and MCF7 cells were sensitive to hormone deprivation but capable of growing in the absence of hormones albeit with a 4- to 6-fold reduced rate. In comparison, ING4 overexpressing T47D cells did not increase in cell numbers in CSS (Figure 1A). 
A

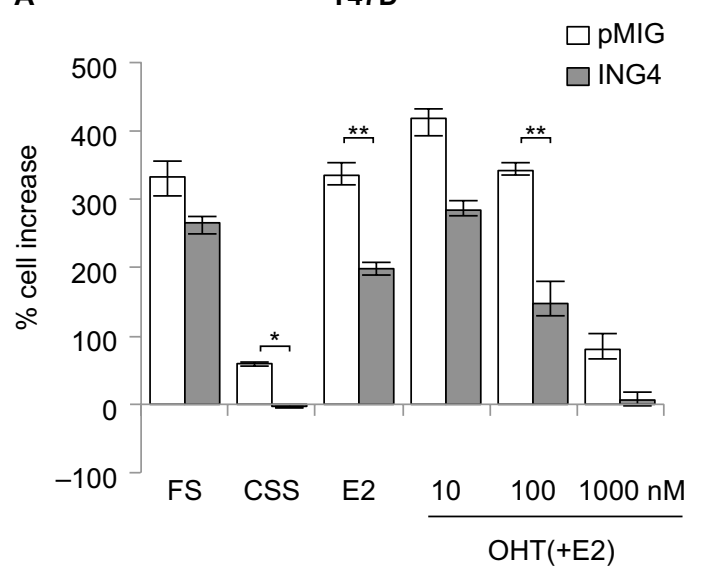

B

MCF7

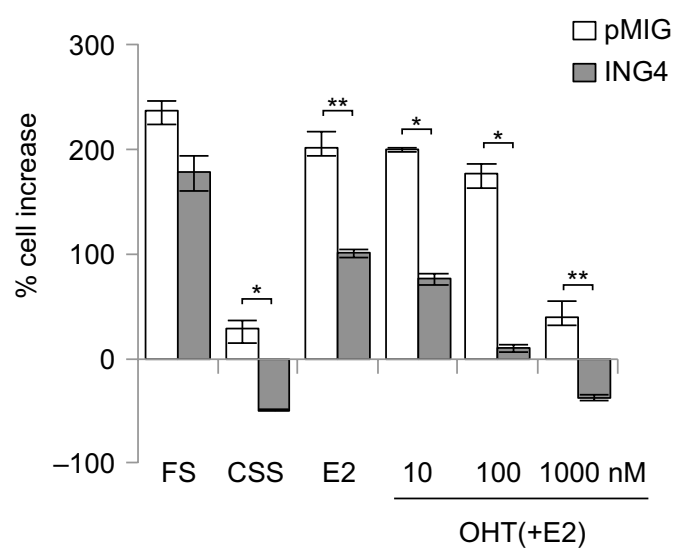

Figure I ER+ breast cancer cells overexpressing ING4 are more sensitive to hormone deprivation and to tamoxifen treatment.

Notes: T47D (A) or MCF7 (B) cells ectopically expressing the PMIG vector or ING4 were hormone-deprived in media containing CSS for 3 days (day 0, d0), followed by 7 days in media containing FS, CSS, CSS+10 nM E2, or CSS+E2 in the presence of OHT in 10, I00 nM, or I $\mu$ M. Cell growth was assessed by SRB colorimetric assay. * $p$-value $<0.001$, ** $p$-value $<0.002$. Error bars represent minimal and maximal values.

Abbreviations: ER+, estrogen receptor-positive; ING4, inhibitor of growth 4; CSS, charcoal-stripped serum; FS, full serum; E2, I7 $\beta$-estradiol; OHT, 4-hydroxy tamoxifen; SRB, sulforhodamine B.
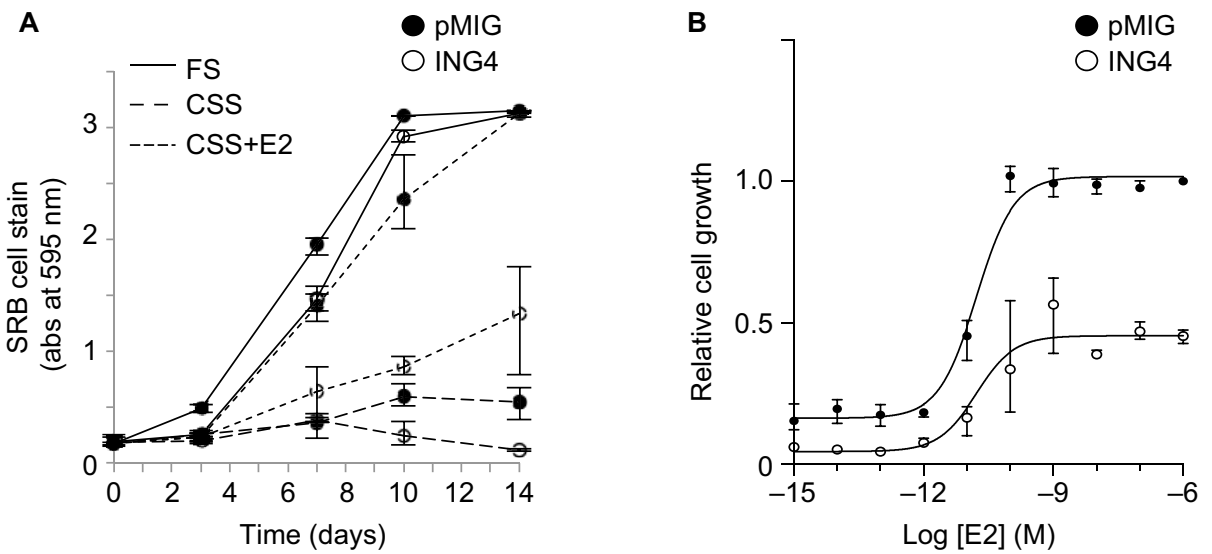

Figure 2 ING4 retards estrogen-dependent growth of T47D cells.

Notes: (A) T47D cells ectopically expressing the pMIG vector or ING4 were grown in media containing FS, CSS, or CSS with 10 nM E2 for I4 days. Cells were fixed at each time point and relative cell numbers were determined by SRB colorimetric assay. pMIG, closed circle; ING4, open circle; FS, solid lines; CSS, long serrated lines; CSS+I0 nM E2, short serrated lines. (B) Ectopic expression of ING4 did not affect EC50 but reduced minimal and maximal effective concentration of E2 for growth. Cells were grown in media containing CSS and various concentrations of E2 for 10 days. Cell growth was assessed by SRB assay and used to generate an EC50 curve. All values are normalized to the values of PMIG treated with I $\mu$ ME2 (maximum E2 concentration).

Abbreviations: ING4, inhibitor of growth 4; CSS, charcoal-stripped serum; FS, full serum; E2, I7 $\beta$-estradiol; SRB, sulforhodamine B; EC50, half-maximal effective concentration.

Moreover, MCF7 cells overexpressing ING4 showed not only no growth but also cell death by $50 \%$ (Figure $1 \mathrm{~B}$ ). These results indicated that ING4 overexpression resulted in increased sensitivity to hormone deprivation and/or suppressed hormone-independent growth of T47D and MCF7 $\mathrm{ER}+$ breast cancer cells.

In the presence of E2 in CSS, pMIG cells grew with a rate comparable to the cells in FS, indicating that estrogen alone could reconstitute growth signaling in the absence of other hormones (Figure 1A and B). In contrast, ING4 cells had reduced rates of cell growth in the presence of $\mathrm{E} 2 \mathrm{com}-$ pared to pMIG cells, indicating that ING4 overexpression attenuated estrogen signaling for the growth of T47D and MCF7 cells ( $p<0.002$ by Student's $t$-test; Figure 1A and B). Consistent with these findings, ING4 overexpressing cells were more sensitive to OHT compared to pMIG cells (Figure 1A and B). At $1 \mu \mathrm{M}$ concentration of OHT, the cell growths were identical to the ones in CSS for pMIG or ING4 cells, indicating that $10 \mathrm{nM}$ of E2 was effectively blocked by $1 \mu \mathrm{M}$ OHT (Figure 1A and B). These results indicated that ING4 increased the sensitivity of T47D and MCF7 cells to tamoxifen by attenuating estrogen-dependent growth and/or by suppressing hormone-independent growth.

\section{ING4 attenuates estrogen signaling for cell growth without affecting estrogen dose-response}

In order to determine whether growth retardation of ING4 cells in E2 was due to altered estrogen response, T47D cell growth rates were compared between pMIG and ING4 cells 
over a 14-day time period in the presence or absence of E2. The results showed that ING4 cells grew at a similar rate as pMIG cells in FS, whereas ING4 cells grew at a 2-3 times reduced rate in $\mathrm{E} 2$ compared to $\mathrm{pMIG}$ cells $(2.7 \times$ slower rate shown in Figure 2A). These results suggested that ING4 may have suppressed E2 signaling for cell growth, potentially requiring higher dose of E2 to achieve optimal growth signaling. EC50 analysis was carried out to determine whether ING4 cells required a higher dosage of estrogen for growth comparable to pMIG cells. The results showed that the EC50 for estrogen in pMIG cells was $15 \mathrm{pM}$ while it was $32.9 \mathrm{pM}$ for ING4 cells (Figure 2B). The difference between the two EC50 values was not statistically significant, indicating that the estrogen dose-response was comparable between pMIG and ING4 cells. However, the difference between the two dose-response curves was statistically significant $(p<0.0001$ by extra sum of squares $F$-test; Figure $2 \mathrm{~B}$ ), indicating that ING4 attenuated the overall E2-mediated cell growth without affecting estrogen dose-response. These results suggested that ING4 functions as a noncompetitive inhibitor of estrogen signaling, potentially by reducing ER protein levels and/or by inhibiting estrogen-independent ER activity.

\section{ING4 does not affect nuclear ER $\alpha$ protein expression}

To determine whether ING4 overexpression resulted in reduction of the ER $\alpha$ protein, Western blot analysis was used. T47D or MCF7 cells expressing pMIG or ING4 were incubated in FS, CSS, CSS+E2, or CSS+E2+OHT, for $24 \mathrm{~h}$. Cells were also treated with fulvestrant ICI182,780 (ICI) in CSS+E2 media for $24 \mathrm{~h}$. Cell lysates were fractionated to nuclear and cytosolic portions and blotted for ER $\alpha$. The results showed that the amounts of the ER $\alpha$ protein in the nuclear fraction were virtually identical between pMIG and ING4 cells (Figure 3A). ER $\alpha$ protein levels in the nuclear fraction decreased when cells were deprived of hormones (CSS), while they increased in the presence of E2, reflecting that ING4 did not influence nuclear localization of ER $\alpha$ upon E2 binding. OHT increased nuclear accumulation of ER $\alpha$, whereas ICI treatment reduced amounts of the ER $\alpha$ protein via enhanced degradation as described previously. ${ }^{28}$ These results demonstrated that ING4 did not reduce nuclear ER $\alpha$ protein levels or nuclear localization of ligand-bound ER $\alpha$, suggesting that ING4 may inhibit the ER activity instead.

It is notable that the ER $\alpha$ protein levels in the cytosolic fractions were reduced by $\sim 2$-fold in T47D cells overexpressing ING4, which was also reflected in the total lysate (Figure 3A). However, E2-induced phosphorylated ERK was comparable between pMIG and ING4 cells, indicating that the

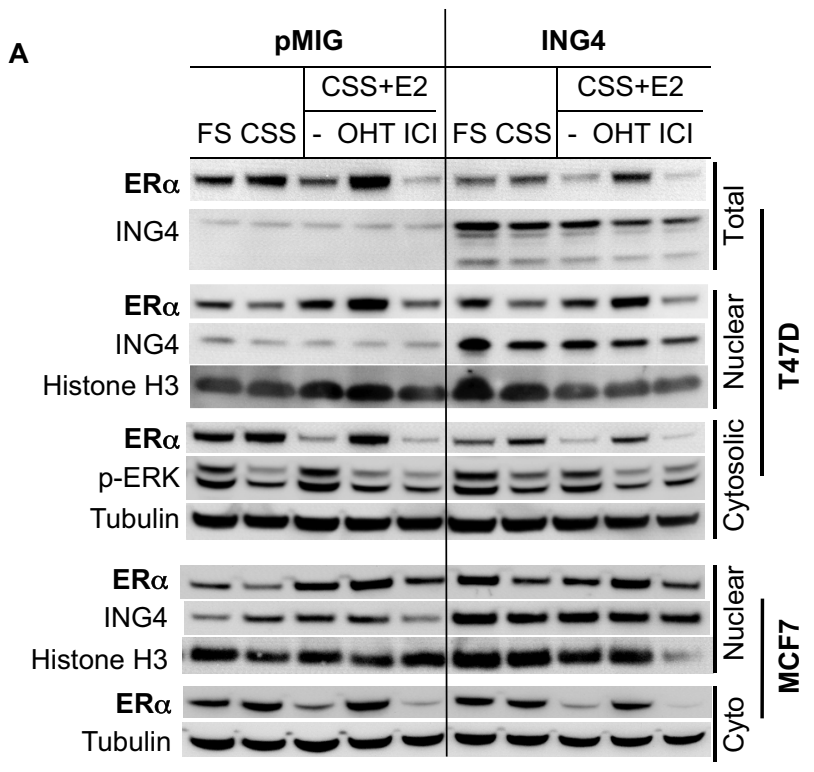

B

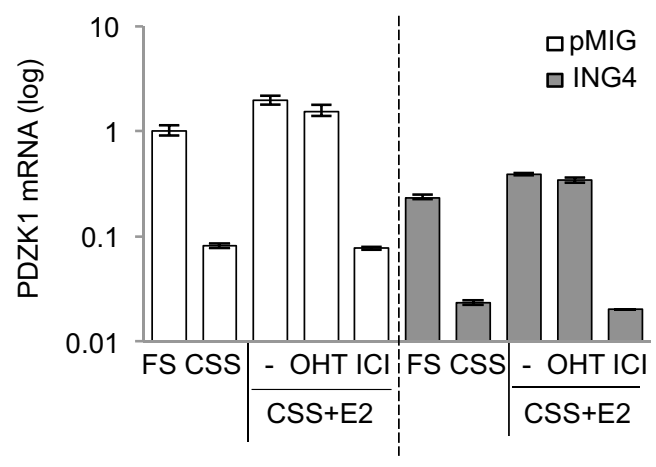

Figure 3 ING4 inhibits $E R \alpha$ transcription activity without affecting $E R \alpha$ protein expression.

Notes: (A) Amounts of the nuclear ER $\alpha$ protein were comparable between the PMIG vector and ING4 expressing T47D or MCF7 cells. Cells were treated for $24 \mathrm{~h}$ with FS, CSS, CSS+10 nM E2, CSS+10 nM E2+I $\mu$ MOHT, or CSS+10 nM E2+100 nM ICII82,780. Total lysate, cytosolic, and nuclear fractions were analyzed by Western blot with antibodies against ING4, ER $\alpha$, or phospho-ERK. Antibodies against histone H3 (nuclear) and $\alpha$-tubulin (cytosolic) were used for loading control. (B) ING4 inhibits the expression of an ER-target gene, PDZKI. PDZKI mRNA levels in the cells treated with the same assay conditions in (A) were quantified by qRTPCR normalized to GAPDH as sample control. Relative expression was normalized to PMIG (FS) samples. “-” represents no OHT or ICl added.

Abbreviations: ING4, inhibitor of growth 4; CSS, charcoal stripped serum; FS, full serum; E2, I7 $\beta$-estradiol; OHT, 4-hydroxy tamoxifen; ICl, ICII82,780; ER, estrogen receptor; qRTPCR, quantitative reverse transcription polymerase chain reaction; ERK, extracellular signal-regulated kinase.

non-genomic function of ER involving the MAPK signaling pathway in the cytosol was not compromised (Figure 3A). In addition, MCF7 cells overexpressing ING4 showed comparable amounts of the ER $\alpha$ protein in the cytosolic and nuclear fractions to the pMIG control cells (Figure 3A, bottom panel). Thus, it is unlikely that ING4 regulates ER $\alpha$ protein expression, and that the reduction in cytoplasmic $E R \alpha$ contributes to the increased sensitivity of T47D-ING4 cells to hormone deprivation. 


\section{ING4 represses an ER-target gene, PDZKI}

As the Westen blot results showed that the amounts of nuclear ER $\alpha$ protein were comparable between pMIG and ING4 cells, ER-mediated transcription was investigated next, by measuring. mRNA expression of one of the ER-target genes, $P D Z K 1$, in T47D cells incubated in the same media conditions of the Western blot samples in Figure 3A, using quantitative RTPCR assays. The results showed that in $\mathrm{pMIG}$ cells, PDZK1 expression increased by 12 -fold and 24-fold in FS and E2, respectively, compared to the cells in CSS (Figure 3B; open bars). Unexpectedly, E2-induced PDZK1 expression was not inhibited by OHT, but was completely inhibited by ICI (Figure 3B). These results suggested that $P D Z K 1$ expression may largely be mediated by E2-independent ER.

In ING4 cells, PDZK1 expression was increased by 10 -fold and 17-fold in FS and E2, respectively, compared to the cells in CSS (Figure 3B; solid bars), indicating that ING4 did not affect E2-induced expression of PDZK1. As was in pMIG cells, E2-induced expression of $P D Z K 1$ was inhibited by ICI but not by OHT in ING4 cells, consistent with E2-independent ER-mediated expression of $P D Z K 1$. It was apparent however, that $P D Z K 1$ expression levels were reduced in ING4 cells in all media conditions by 4 - to 5 -fold, compared to the ones in pMIG cells (Figure 3B). These results indicated that ING4 repressed "basal level" expression of $P D Z K 1$, potentially by inhibiting genomic action of E2-independent ER.

\section{Fulvestrant, not tamoxifen, inhibits the basal PDZKI expression}

Next was tested whether PDZK1 expression in pMIG cells could be reduced to the level in ING4 cells by inhibiting ER.
pMIG cells were treated in FS, CSS, or CSS+E2, and PDZK1 expression was compared in the presence or absence of tamoxifen (OHT) or fulvestrant (ICI), compared to the PDZK1 expression in ING4 cells. The results showed that OHT did not, but ICI did reduce PDZK1 expression in FS and E2, suggesting that $P D Z K 1$ expression was E2-independent but ER-dependent (Figure 4A). In FS, ING4 reduced the expression level of $P D Z K 1$ comparable to ICI-treated pMIG cells, indicating that ING4 too inhibited E2-independent ER activity (Figure 4A). In E2, ING4 reduced PDZK1 expression but to a lesser degree compared to ICI-treated pMIG cells, suggesting that E2 may override ING4-mediated PDZK1 repression (Figure 4A). In CSS, ICI treatment did not further reduce $P D Z K 1$ expression, suggesting that the "basal level" expression of $P D Z K 1$ may be ER-independent. In contrast, ING4 reduced $P D Z K 1$ expression in CSS, suggesting that ING4 may additionally inhibit ER-independent expression of $P D Z K 1$ (see "Discussion"). It is notable that OHT increased $P D Z K 1$ expression in CSS, indicating an agonist effect of OHT in the absence of E2 (Figure 4A). Collectively, these results demonstrated that while OHT did not, both ICI and ING4 decreased PDZK1 expression, indicating that ING4 inhibited E2-independent ER.

\section{Fulvestrant, but not tamoxifen, inhibits hormone-independent cell growth of T47D cells}

As ING4 inhibited E2-independent ER activity that mediated PDZK1 expression, it was investigated whether ING4 inhibition of E2-independent cell growth was ER-dependent. pMIG cell growth in CSS in the presence of OHT or ICI was
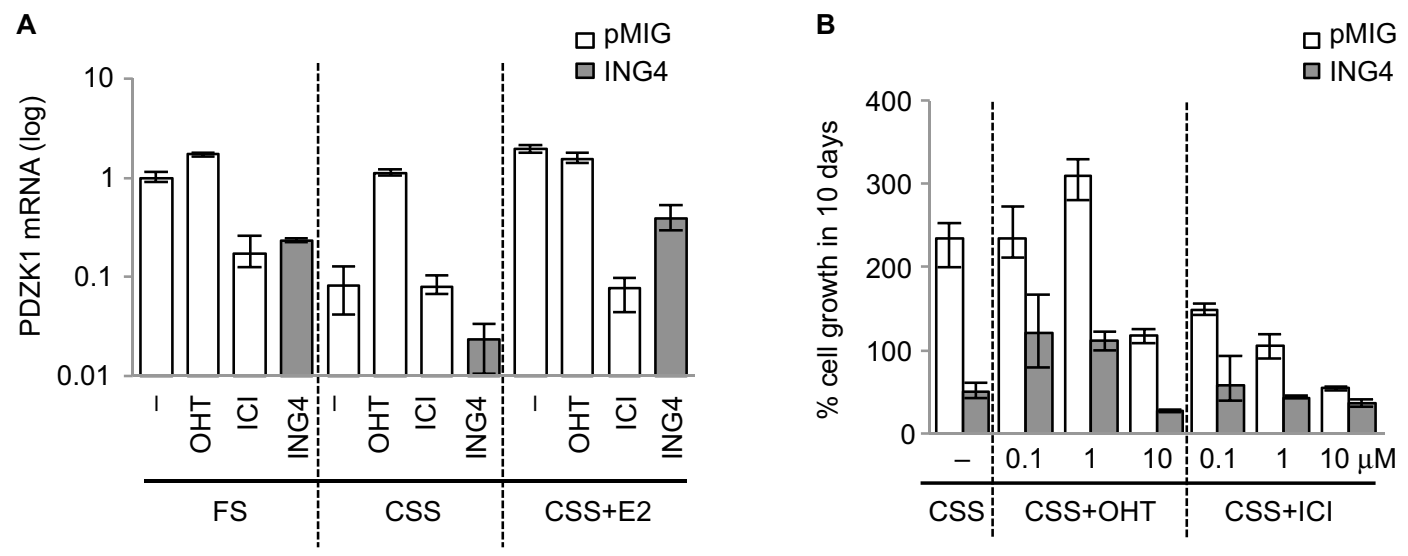

Figure 4 ING4 inhibits ligand-independent ER activity.

Notes: (A) ICl treatment, not OHT, of the vector pMIG expressing T47D cells results in diminished PDZKI expression comparable to ING4 expressing cells. Cells were treated with I $\mu \mathrm{MOHT}$ or I $\mu \mathrm{M} \mathrm{ICl}$ in FS, CSS, or CSS+E2, for $24 \mathrm{~h}$ and harvested for total RNA isolation. PDZKI mRNA was quantified by qRTPCR, normalized to GAPDH. (B) ICl, not OHT, suppresses hormone-independent growth of PMIG expressing T47D cells in a dose-dependent manner. Cells were grown in CSS media for I0 days with OHT in incremental concentrations of $100 \mathrm{nM}$, I, or $10 \mu \mathrm{M}$, or with ICl in incremental concentrations of $100 \mathrm{nM}$, I, or $10 \mu \mathrm{M}$. “-” represents no OHT or ICl added.

Abbreviations: ING4, inhibitor of growth 4; CSS, charcoal stripped serum; FS, full serum; E2, I7 $\beta$-estradiol; OHT, 4-hydroxy tamoxifen; ER, estrogen receptor; ICl, ICII82,780; qRTPCR, quantitative reverse transcription polymerase chain reaction. 
compared to ING4 cell growth. The results showed that pMIG cell number increased $200 \%$ in 10 days in CSS, demonstrating hormone-independent growth (Figure 4B). In contrast, ING4 cell number was decreased to $50 \%$, indicating cell death in prolonged hormone deprivation conditions (Figure 4B). Next, increasing log doses of OHT or ICI were used in CSS and the percent cell growth of pMIG cells was compared to ING4 cell growth in CSS. The results showed that OHT at 0.1 or $1 \mu \mathrm{M}$ concentration did not inhibit pMIG cell growth in CSS. The same concentrations of OHT stimulated the growth of ING4 cells in CSS, indicating an agonist effect of OHT in cell growth in the absence of E2. These findings are consistent with the results showing that $\mathrm{OHT}$ induced $P D Z K 1$ expression in the absence of E2 (Figure 4A). In the presence of $10 \mu \mathrm{MOHT}$, both pMIG and ING4 cells showed cell death, reflecting a cytotoxic effect of $\mathrm{OHT}$ at a high concentration (Figure 4B). In contrast, ICI inhibited growth of pMIG cells in a dose-dependent manner, with $10 \mu \mathrm{M}$ concentration effectively suppressing hormoneindependent growth of pMIG cells to the level of ING4 cells (Figure 4B). These results demonstrated that ICI but not OHT rendered pMIG cells sensitive to hormone-deprivation as ING4 cells. These results support the conclusion that ING4 inhibits E2-independent ER activity that allows ER+ breast cancer cells to grow in the absence of estrogen.

\section{ING4 inhibits the expression of estrogen responsive element (ERE)-mediated reporter luciferase gene}

Next was investigated whether ING4 regulation of ER-target genes involved the ERE sequence, a canonical ER binding site. The pGL2-ERE plasmid containing a luciferase reporter gene with ERE in the promoter region was used to establish T47D-pMIG or T47D-ING4 cell lines stably transfected the reporter construct. Luciferase assay was carried out using cell lysates harvested after incubating cells in CSS for 2 days and additional $24 \mathrm{~h}$ with or without E2. The results showed that ING4 cells contained at least 100-fold less luciferase activity in CSS (Figure 5A) compared to pMIG cells, indicating that ING4 repressed basal expression of the luciferase reporter gene. E2 treatment induced luciferase activity by 5 -fold in pMIG cells but failed to induce it in ING4 cells, indicating that ING4 inhibited E2-induced expression of the reporter gene (Figure 5A). Thus, it was concluded that ING4 inhibited both basal and E2-induced expression of the luciferase reporter gene facilitated by the ERE sequence.

It was notable that transient transfection of the reporter construct did not yield any discernable or reproducible differences in the basal or E2-induced luciferase expression between pMIG and ING4 cells (data not shown). These results indicated that the action of ING4 on transcription modulation required the reporter gene integration in the genome as has been reported previously, consistent with the proposed mechanism of ING4 as a chromatin remodeler. ${ }^{25}$

\section{ING4 represses selective ER-target gene expression}

ING4 regulation of four endogenous ER-target gene expression was next investigated: $P D Z K 1, T F F 1, M Y C$, and $P G R$. The first two genes, $P D Z K 1$ and $T F F 1$, have been shown to contain a canonical ERE in their promoter regions while the latter two, $M Y C$ and $P G R$, have a putative ERE half site. ${ }^{29-32}$ The results showed that PDZK1 or TFF1 mRNA levels were 10 -fold less in ING4 cells in all media conditions compared to pMIG cells (Figure 5B). In contrast, ING4 did not affect $M Y C$ or $P G R$ expression (Figure 5B). E2-induced fold expression of all four genes was not or nominally affected by ING4 (Figure 5B). E2-induced expression of MYC seemed to be reduced in ING4 cells at a 72-h time point (Figure 5B). However, a time course experiment showed comparable MYC induction in ING4 cells at a later time point (data not shown). It is unclear whether ING4 plays a role in temporal regulation of $M Y C$ expression. Nevertheless, these results indicated that ING4 repressed basal expression of $P D Z K 1$ and $T F F 1$, but not of $M Y C$ and $P G R$ (Figure 5B). Although limited in scope, since both $P D Z K 1$ and TFF1 in addition to the luciferase reporter gene contain the canonical ERE sequence in their promoter regions, these findings may suggest that ING4 regulates basal expression of ER-target genes that contain ERE.

\section{Discussion}

The present study has demonstrated that ING4 inhibits estrogen-independent ER activity that sustains growth of $\mathrm{ER}+$ breast cancer cells under hormone-deprived conditions. These results suggest that breast tumors that have downregulated ING4 conversely contain high estrogenindependent ER activity. Consequently, antiestrogen agents such as tamoxifen and AIs would not be effective in the treatment of ING4-low ER+ breast cancer. These findings provide a possible explanation for the previous report that breast cancer patients with ING4-low tumors recur with faster rates despite adjuvant tamoxifen therapy. ${ }^{25}$ The findings further suggest that blocking estrogen with SERM or AI is not sufficient for the treatment of ING4-low ER+ 
A

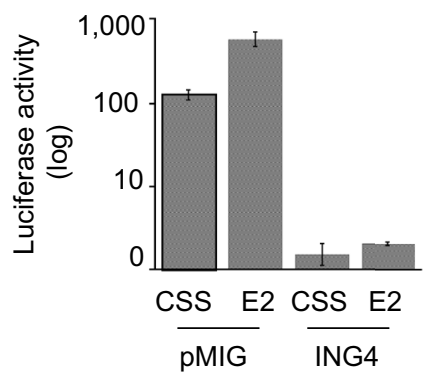

B
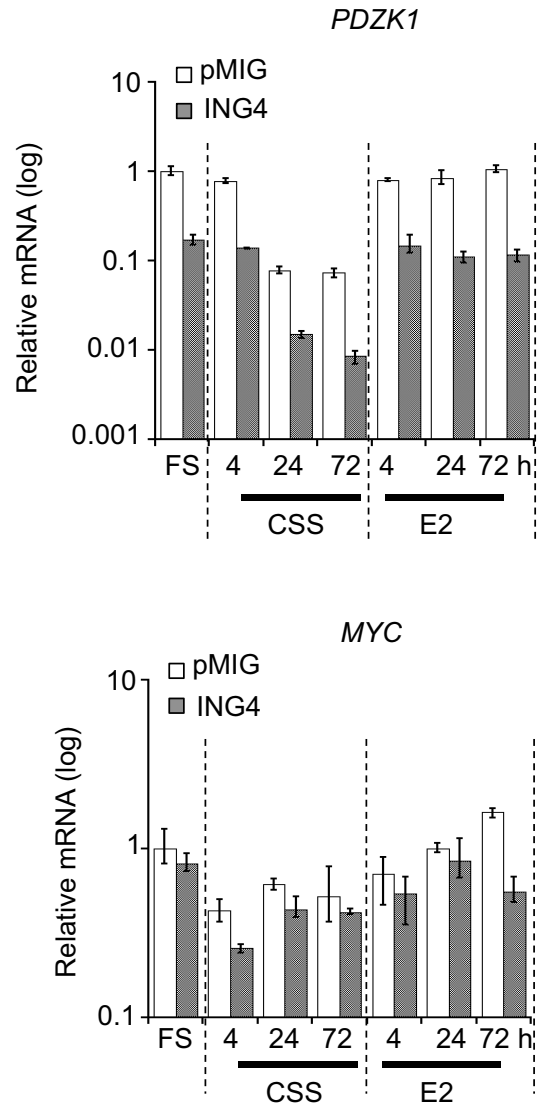
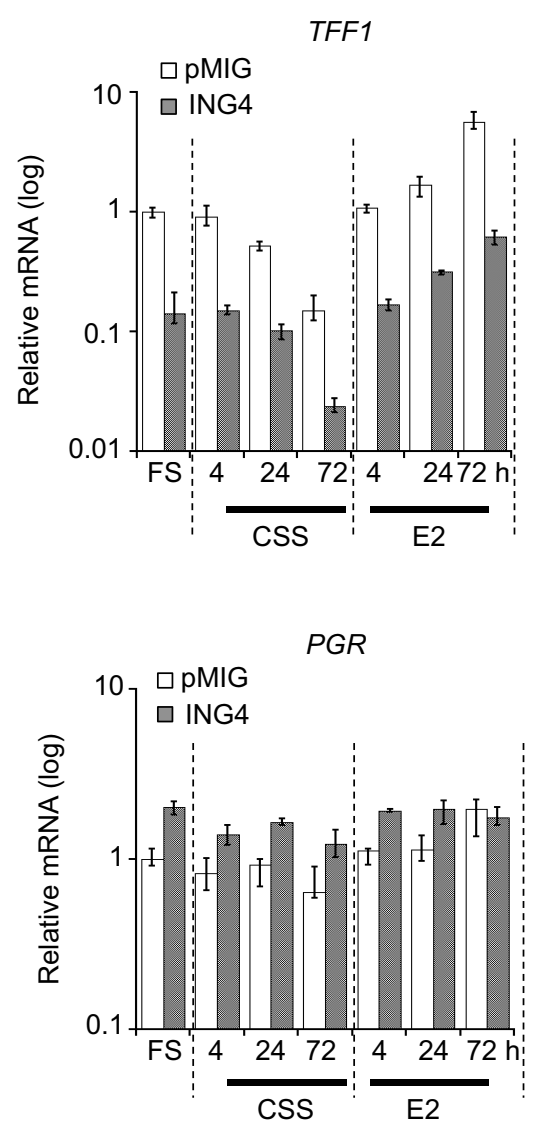

Figure 5 ING4 represses transcription of selective ER-target genes containing ERE.

Notes: (A) Expression of a luciferase reporter construct containing an ERE promoter sequence in T47D cells. Cells were treated with CSS (-) for 2 days and additional $24 \mathrm{~h}$ with (E2) or without (-) $10 \mathrm{nM}$ E2. (B) Relative mRNA expression of the ER-target genes, TFFI, PDZKI, MYC, and PGR in cells treated with CSS (-E2) or I0 nM E2 (+E2) for 4, 24, or $72 \mathrm{~h}$.

Abbreviations: ING4, inhibitor of growth 4; CSS, charcoal stripped serum; FS, full serum; E2, I7 $\beta$-estradiol; ER, estrogen receptor; ERE, estrogen response element.

breast cancer, but selective ER degraders such as fulvestrant that facilitates ER degradation may be more effective in preventing tumor recurrence. Supportive of this idea are the recent clinical trials of fulvestrant showing improved patient survival over AI. ${ }^{33}$ Whether breast tumors with low ING4 levels show increased response to fulvestrant has not been determined.

The study results put forth ING4 downregulation resulting in disregulated estrogen-independent ER activity as a mechanism for antiestrogen therapy resistance. High levels of ligand-independent ER activity have been detected in
AI-resistant breast cancer cells selected in vitro or in patient tumor samples collected after the treatment. ${ }^{34,35}$ These studies have suggested that ligand-independent ER activity may be an acquired trait under therapy selection. Whether increased ligand-independent ER activity in AI recurrent tumors is in part due to ING4 downregulation is not known, nor is ING4 downregulation an acquired trait under therapy selection. However, it was previously shown that ING4 expression in primary tumors could stratify recurrent patients with tamoxifen therapy, ${ }^{25}$ indicating that ING4 downregulation had occurred prior to tamoxifen therapy at least in this patient 
cohort. These results suggest that low ING4 expression may confer intrinsic tamoxifen resistance prior to therapy and further suggest that ING4 expression in primary tumors could be used as a prognostic biomarker predictive of antiestrogen therapy response.

The molecular mechanism of ING4 downregulation in tumors is not well understood. The previous study reported that $16 \%$ of primary breast tumors harbor a single copy ING4 gene deletion, which is likely to account for low ING4 expression in a subset of tumors. ${ }^{24}$ Other mechanisms including epigenetic regulation of ING4 gene expression are currently unknown. Such mechanisms may be potentially targeted by pharmacological means to achieve ING4 induction in order to thereby reduce ligand-independent ER activity and prevent tumor recurrence in breast cancer.

In this study, ING4 was shown to repress the expression of selective ER-target genes, PDZK1 and TFF1, but not $M Y C$ and $P G R$. Whether ING4-repressed ER-target genes including $P D Z K 1$ and $T F F 1$ play a functional role in the pathogenesis and/or therapy resistance of ER+ breast cancer is presently unclear. It is notable, however, that $P D Z K 1$ has been identified as a breast cancer susceptibility gene in genome-wide association studies. ${ }^{36}$ Moreover, ectopic expression of $P D Z K 1$ has been shown to increase the proliferation of MCF7 ER+ breast cancer cells in the presence or absence of estrogen, suggesting an oncogenic potential of PDZK $1 .{ }^{37}$ Thus, repression of $P D Z K 1$ basal expression could be part of ING4's tumor suppressive mechanism. However, PDZKI mRNA expression levels alone did not stratify recurrent breast cancer patients with tamoxifen therapy (data not shown), indicating that $P D Z K 1$ regulation is not the sole tumor suppressor function of ING4 in breast cancer and/or therapy response.

The scope of ER-target genes that ING4 regulates is currently unclear, as several gene expression-profiling studies have not yielded a consistent model of ING4 action (S Kim, unpublished data). This is likely due to the dynamic nature of $E R \alpha$ transcriptional activity reported in extensive and numerous studies, identifying 300 to $>1,000$ estrogeninduced ER-target genes. ${ }^{3,38,39}$ Moreover, studies have identified diverse co-activators and repressors that interact with liganded and unliganded ER $\alpha .^{40-43}$ The exact molecular mechanism of ING4 in ER $\alpha$ regulation is presently unclear. ING4 and ER $\alpha$ did not co-immunoprecipitate, suggesting indirect and/or transient interactions if any ( $\mathrm{S} \mathrm{Kim,}$ unpublished data). Limited chromatin immunoprecipitation (ChIP) experiments showed that $\mathrm{ER} \alpha$ was present on the ERE promoter sequence of the reporter construct in ING4 overexpressing cells, indicating that ING4 did not inhibit $\mathrm{ER} \alpha$ binding to the genome (S Kim, unpublished data). However, whether ING4 binds to the same ERE promoter region is undetermined because no evidence is available to confirm that the ING4 antibody is capable of ChIP. Such an antibody will be a crucial reagent to decipher the ING4 mechanism.

Recent studies have also shown that unliganded ER $\alpha$ binds to 4,000-5,000 sites in the genome. ${ }^{35,40,44}$ Caizzie et al in their study demonstrated that unliganded ER $\alpha$ regulates 900 genes that largely overlap with the set of estrogeninduced ER-target genes, suggesting that unliganded ER $\alpha$ may maintain the basal expression of estrogen-inducible ER-target genes. ${ }^{40}$ These results support the current study findings that basal expression of PDZK1 was effectively repressed by fulvestrant but not by tamoxifen. The current study also suggests that ING4 may play a role in the regulation of genes that contain canonical ERE in their promoter regions. To the authors' knowledge, this study puts forth ING4 as the first negative regulator of unliganded ER $\alpha$. Caizzi et al also reported that unliganded ER $\alpha$ regulated expression of a distinct set of genes involved in the luminal epithelial cell fate. ${ }^{40}$ These data would suggest that ING4 may also play a role in breast epithelial differentiation via regulation of unliganded ER $\alpha$. Supportive of this, expression of a dominant negative mutant of ING4 resulted in hyperplastic abnormal ductal structure in mouse mammary glands and cooperated with the MYC oncogene to form mammary tumors. ${ }^{45}$ In addition, ING4 was shown to regulate gene transcription during prostate epithelial cell differentiation and that in the absence of ING4, primary prostate epithelial cells progress to cancer with additional oncogenic events. ${ }^{46}$ Taken together, it can be postulated that ING4 plays a broader transcriptional regulatory role in epithelial differentiation and/or maintenance, thus suppressing tumorigenesis. A more direct approach to determine ING4-target genes and/ or ING4 interaction with unliganded ER $\alpha$ in the genome in mammary tissue development will likely clarify the roles of ING4/ER $\alpha$ interplay in mammary epithelial cell differentiation and breast cancer.

Finally, it is notable that low expression of ING4 has been correlated with advanced tumors, poor patient outcome, and therapy resistance in diverse cancers including glioma, head and neck squamous carcinoma, breast cancer, lung cancer, hepatocellular carcinoma, prostate cancer, and multiple myeloma. ${ }^{23}$ These studies attest to a tumor suppressive function of ING4 in many tissue types, not all of which express $\mathrm{ER} \alpha$. Therefore, inhibition of unliganded $\mathrm{ER} \alpha$ may represent 
one of multiple functions of ING4. Indeed, ING4 has been shown to regulate other transcriptional programs related to p53 in DNA damage response, NF $\kappa$ B in tumor progression and immune response, and Myc in prostate cell differentiation and mammary tumorigenesis. ${ }^{23}$ The molecular mechanism of ING4 that enables its interactions with diverse transcriptional factors including unliganded ER $\alpha$ will need to be determined in order to address the fundamental function and mechanism of ING4 in normal and cancer cell development and also to explore a potential innovative strategy to treat cancer.

\section{Acknowledgments}

The authors thank Dr Sara Byron for her critical reading of the manuscript and helpful discussions. The current address of Madeline M Keenen is University of California, San Francisco, CA, USA (Email madeline.keenen@ucsf.edu). This work was supported by funding from the University of Arizona College of Medicine-Phoenix (to Suwon Kim).

\section{Disclosure}

The authors report no conflicts of interest in this work.

\section{References}

1. Anderson WF, Chatterjee N, Ershler WB, Brawley OW. Estrogen receptor breast cancer phenotypes in the Surveillance, Epidemiology, and End Results database. Breast Cancer Res Treat. 2002;76(1):27-36.

2. Shao W, Brown M. Advances in estrogen receptor biology: prospects for improvements in targeted breast cancer therapy. Breast Cancer Res. 2004;6(1):39-52.

3. Welboren WJ, Sweep FC, Span PN, Stunnenberg HG. Genomic actions of estrogen receptor alpha: what are the targets and how are they regulated? Endocr Relat Cancer. 2009;16(4):1073-1089.

4. Davies C, Godwin J, Gray R, et al; Early Breast Cancer Trialists' Collaborative Group. Relevance of breast cancer hormone receptors and other factors to the efficacy of adjuvant tamoxifen: patient-level meta-analysis of randomised trials. Lancet. 2011;378(9793):771-784.

5. Dowsett M, Forbes JF, Bradley R, et al; Early Breast Cancer Trialists' Collaborative Group. Aromatase inhibitors versus tamoxifen in early breast cancer: patient-level meta-analysis of the randomised trials. Lancet. 2015;386(10001):1341-1352.

6. Osborne CK, Schiff R. Mechanisms of endocrine resistance in breast cancer. Annu Rev Med. 2011;62:233-247.

7. Shou J, Massarweh S, Osborne CK, et al. Mechanisms of tamoxifen resistance: increased estrogen receptor-HER2/neu cross-talk in ER/HER2-positive breast cancer. J Natl Cancer Inst. 2004;96(12): 926-935.

8. Fox EM, Miller TW, Balko JM, et al. A kinome-wide screen identifies the insulin/IGF-I receptor pathway as a mechanism of escape from hormone dependence in breast cancer. Cancer Res. 2011;71(21):6773-6784.

9. Turner N, Pearson A, Sharpe R, et al. FGFR1 amplification drives endocrine therapy resistance and is a therapeutic target in breast cancer. Cancer Res. 2010;70(5):2085-2094.

10. Martin LA, Farmer I, Johnston SR, Ali S, Marshall C, Dowsett M. Enhanced estrogen receptor (ER) alpha, ERBB2, and MAPK signal transduction pathways operate during the adaptation of MCF-7 cells to long term estrogen deprivation. J Biol Chem. 2003;278(33):30458-30468.
11. Miller TW, Hennessy BT, Gonzalez-Angulo AM, et al. Hyperactivation of phosphatidylinositol-3 kinase promotes escape from hormone dependence in estrogen receptor-positive human breast cancer. J Clin Invest. 2010;120(7):2406-2413.

12. Miller TW, Balko JM, Ghazoui Z, et al. A gene expression signature from human breast cancer cells with acquired hormone independence identifies MYC as a mediator of antiestrogen resistance. Clin Cancer Res. 2011;17(7):2024-2034.

13. Lupien M, Meyer CA, Bailey ST, et al. Growth factor stimulation induces a distinct ER(alpha) cistrome underlying breast cancer endocrine resistance. Genes Dev. 2010;24(19):2219-2227.

14. Ma CX, Reinert T, Chmielewska I, Ellis MJ. Mechanisms of aromatase inhibitor resistance. Nat Rev Cancer. 2015;15(5):261-275.

15. Johnston S, Pippen J Jr, Pivot X, et al. Lapatinib combined with letrozole versus letrozole and placebo as first-line therapy for postmenopausal hormone receptor-positive metastatic breast cancer. J Clin Oncol. 2009;27(33):5538-5546.

16. Osborne CK, Neven P, Dirix LY, et al. Gefitinib or placebo in combination with tamoxifen in patients with hormone receptor-positive metastatic breast cancer: a randomized phase II study. Clin Cancer Res. 2011;17(5):1147-1159.

17. Robertson JF, Ferrero JM, Bourgeois H, et al. Ganitumab with either exemestane or fulvestrant for postmenopausal women with advanced, hormone-receptor-positive breast cancer: a randomised, controlled, double-blind, phase 2 trial. Lancet Oncol. 2013;14(3):228-235.

18. Kim S. New and emerging factors in tumorigenesis: an overview. Cancer Manag Res. 2015;7:225-239.

19. Robinson DR, WuYM, Vats P, et al. Activating ESR1 mutations in hormoneresistant metastatic breast cancer. Nat Genet. 2013;45(12):1446-1451.

20. Toy W, Shen $Y$, Won H, et al. ESR1 ligand-binding domain mutations in hormone-resistant breast cancer. Nat Genet. 2013;45(12):1439-1445.

21. Thomas C, Gustafsson JA. Estrogen receptor mutations and functional consequences for breast cancer. Trends Endocrinol Metab. 2015;26(9): $467-476$.

22. Wang P, Bahreini A, Gyanchandani R, et al. Sensitive detection of mono- and polyclonal ESR1 mutations in primary tumors, metastatic lesions, and cell-free DNA of breast cancer patients. Clin Cancer Res. 2016;22(5):1130-1137.

23. Cui S, Gao Y, Zhang K, Chen J, Wang R, Chen L. The emerging role of inhibitor of growth 4 as a tumor suppressor in multiple human cancers. Cell Physiol Biochem. 2015;36(2):409-422.

24. Tapia C, Zlobec I, Schneider S, et al. Deletion of the inhibitor of growth 4 (ING4) tumor suppressor gene is prevalent in human epidermal growth factor 2 (HER2)-positive breast cancer. Hum Pathol. 2011;42(7):983-990.

25. Byron SA, Min E, Thal TS, et al. Negative regulation of NFkappaB by the ING4 tumor suppressor in breast cancer. PLoS One. 2012;7(10):e46823.

26. Kim S, Chin K, Gray JW, Bishop JM. A screen for genes that suppress loss of contact inhibition: identification of ING4 as a candidate tumor suppressor gene in human cancer. Proc Natl Acad Sci U S A. 2004;101(46):16251-16256.

27. Hall JM, McDonnell DP. The estrogen receptor beta-isoform (ERbeta) of the human estrogen receptor modulates ERalpha transcriptional activity and is a key regulator of the cellular response to estrogens and antiestrogens. Endocrinology. 1999;140(12):5566-5578.

28. Lupien M, Jeyakumar M, Hebert E, et al. Raloxifene and ICI182,780 increase estrogen receptor-alpha association with a nuclear compartment via overlapping sets of hydrophobic amino acids in activation function 2 helix 12. Mol Endocrinol. 2007;21(4):797-816.

29. Tachibana K, Anzai N, Ueda C, et al. Regulation of the human PDZK1 expression by peroxisome proliferator-activated receptor alpha. FEBS Lett. 2008;582(28):3884-3888.

30. Sewack GF, Hansen U. Nucleosome positioning and transcriptionassociated chromatin alterations on the human estrogen-responsive pS2 promoter. J Biol Chem. 1997;272(49):31118-31129.

31. Dubik D, Shiu RP. Mechanism of estrogen activation of c-myc oncogene expression. Oncogene. 1992;7(8):1587-1594. 
32. Petz LN, Ziegler YS, Schultz JR, Kim H, Kemper JK, Nardulli AM. Differential regulation of the human progesterone receptor gene through an estrogen response element half site and $\mathrm{Sp} 1$ sites. J Steroid Biochem Mol Biol. 2004;88(2):113-122.

33. Robertson JF, Llombart-Cussac A, Rolski J, et al. Activity of fulvestrant $500 \mathrm{mg}$ versus anastrozole $1 \mathrm{mg}$ as first-line treatment for advanced breast cancer: results from the FIRST study. J Clin Oncol. 2009;27(27): 4530-4535.

34. Miller TW, Balko JM, Fox EM, et al. ERalpha-dependent E2F transcription can mediate resistance to estrogen deprivation in human breast cancer. Cancer Discov. 2011;1(4):338-351.

35. Vareslija D, McBryan J, Fagan A, et al. Adaptation to AI therapy in breast cancer can induce dynamic alterations in ER activity resulting in estrogen-independent metastatic tumors. Clin Cancer Res. 2016; 22(11):2765-2777.

36. Michailidou K, Beesley J, Lindstrom S, et al. Genome-wide association analysis of more than 120,000 individuals identifies 15 new susceptibility loci for breast cancer. Nat Genet. 2015;47(4):373-380.

37. Kim H, Abd Elmageed ZY, Ju J, et al. PDZK1 is a novel factor in breast cancer that is indirectly regulated by estrogen through IGF-1R and promotes estrogen-mediated growth. Mol Med. 2013;19:253-262.

38. Carroll JS, Meyer CA, Song J, et al. Genome-wide analysis of estrogen receptor binding sites. Nat Genet. 2006;38(11):1289-1297.
39. Lin CY, Vega VB, Thomsen JS, et al. Whole-genome cartography of estrogen receptor alpha binding sites. PLoS Genet. 2007;3(6):e87.

40. Caizzi L, Ferrero G, Cutrupi S, et al. Genome-wide activity of unliganded estrogen receptor-alpha in breast cancer cells. Proc Natl Acad Sci U S A. 2014;111(13):4892-4897.

41. Shang Y, Hu X, DiRenzo J, Lazar MA, Brown M. Cofactor dynamics and sufficiency in estrogen receptor-regulated transcription. Cell. 2000;103(6):843-852.

42. Metivier R, Penot G, Hubner MR, et al. Estrogen receptor-alpha directs ordered, cyclical, and combinatorial recruitment of cofactors on a natural target promoter. Cell. 2003;115(6):751-763.

43. Carroll JS, Liu XS, Brodsky AS, et al. Chromosome-wide mapping of estrogen receptor binding reveals long-range regulation requiring the forkhead protein FoxA1. Cell. 2005;122(1):33-43.

44. Stellato C, Porreca I, Cuomo D, Tarallo R, Nassa G, Ambrosino C. The "busy life" of unliganded estrogen receptors. Proteomics. 2016; 16(2):288-300.

45. Kim S, Welm AL, Bishop JM. A dominant mutant allele of the ING4 tumor suppressor found in human cancer cells exacerbates MYC-initiated mouse mammary tumorigenesis. Cancer Res. 2010;70(12):5155-5162.

46. Berger PL, Frank SB, Schulz VV, et al. Transient induction of ING4 by Myc drives prostate epithelial cell differentiation and its disruption drives prostate tumorigenesis. Cancer Res. 2014;74(12):3357-3368.
Breast Cancer - Targets and Therapy

\section{Publish your work in this journal}

Breast Cancer - Targets and Therapy is an international, peerreviewed open access journal focusing on breast cancer research, identification of therapeutic targets and the optimal use of preventative and integrated treatment interventions to achieve improved outcomes, enhanced survival and quality of life for the cancer patient

\section{Dovepress}

The manuscript management system is completely online and includes a very quick and fair peer-review system, which is all easy to use. Visit http://www.dovepress.com/testimonials.php to read real quotes from published authors. 